Revue française de la traduction

\title{
Emprunts du français et de l'allemand au latin. Vrais et faux amis
}

\section{Michel Corbellari}

\section{(2) OpenEdition}

Journals

\section{Édition électronique}

URL : http://journals.openedition.org/traduire/218

DOI : $10.4000 /$ traduire.218

ISSN : 2272-9992

\section{Éditeur}

Société française des traducteurs

\section{Édition imprimée}

Date de publication : 1 juin 2011

Pagination : 121-128

ISSN : 0395-773X

\section{Référence électronique}

Michel Corbellari, « Emprunts du français et de l'allemand au latin. Vrais et faux amis », Traduire [En ligne], 224 | 2011, mis en ligne le 03 février 2014, consulté le 19 avril 2019. URL : http:// journals.openedition.org/traduire/218; DOI : 10.4000/traduire.218 


\section{Emprunts du français et de l'allemand au latin Vrais et faux amis}

\section{Michel Corbellari}

L'étude comparée des latinismes en français et en allemand nous amène à distinguer plusieurs cas :

\section{Mots ou expressions qui existent dans les deux langues et ont le même sens}

lls sont nombreux.

\begin{tabular}{|l|l|}
\hline das Alter Ego & l'alter ego \\
\hline der Cannabis & le cannabis \\
\hline der Deus ex machina & le deus ex machina \\
\hline das Exvoto & l'ex-voto \\
\hline das Imprimatur & l'imprimatur \\
\hline der Index & l'index (également : l'indice) \\
\hline der Malus & le malus \\
\hline die Mater dolorosa & la mater dolorosa \\
\hline der Modus Operandi & le modus operandi (également : le mode opératoire) \\
\hline die Parabellum & le parabellum \\
\hline die Persona non grata & la persona non grata \\
\hline das Plenum & le plénum \\
\hline das Postskriptum & le post-scriptum \\
\hline der Tonus & le tonus \\
\hline die Vox Populi & la vox populi \\
\hline ad usum Delphini & ad usum delphini \\
\hline
\end{tabular}

ad infinitum, ad libitum, ad nauseam, bis !, ergo, ex abrupto, in extenso, in medias res, in situ, ipso facto, mutatis mutandis, nolens volens, post mortem, sic, via, etc. 


\section{Remarques}

1. die Libido/la libido est un mot créé par Freud à partir du latin libido, le désir.

2. Si l'on trouve ibidem dans les deux langues, les abréviations sont différentes : ib. ou ibd. en allemand, ibid. en français.

3. Der/das Rebus vient bien du latin, mais par l'intermédiaire du français.

4. Certains " latinismes " sont des créations récentes : die Pax Americana/la pax americana, der Homo oeconomicus/l'homo oeconomicus

5. Notons la différence entre der Status quo et le statu quo, das Nonplusultra et le nec plus ultra, der Infarkt et l'infarctus, das Quidproquo et le quiproquo. (N.B. Au sens français de quiproquo, l'allemand Quidproquo est vieilli).

6. Versus s'abrège vs. (avec point) en allemand et vs (sans point) en français.

7. A die Korrigenda correspond les errata, à l'allemand stante pede, le français illico (fam.), à l'allemand per pedes le français pedibus (fam.) ; quant à pedibus cum jambis (fam.) il est évidemment réservé au français.

8. Die Villa/la villa vient du latin par l'intermédiaire de l'italien.

9. Der Sponsor/le sponsor, das Tandem/le tandem, versus viennent du latin par l'intermédiaire de l'anglais.

10. Le parabellum vient du latin (si vis pacem, para bellum) par l'intermédiaire de l'allemand.

11. Le bis se dit das Dacapo.

12. Extra muros et intra muros s'écrivent sans trait d'union en allemand et avec trait d'union en français : extra-muros, intra-muros.

\section{Mots dont la forme française a été " francisée ", voire " traduite "}

Ils sont très nombreux. 


\begin{tabular}{|c|c|}
\hline der Advocatus Diaboli & l'avocat du diable \\
\hline das Bestiarium & le bestiaire \\
\hline der Circulus vitiosus & le cercle vicieux \\
\hline die Conditio humana & la condition humaine \\
\hline das Corpus delicti & le corps du délit \\
\hline das Evangelium & l'Évangile \\
\hline der Exodus & l'exode \\
\hline das Fluidum & le fluide \\
\hline der Genius Loci & le génie du lieu \\
\hline der Homunkulus & I'homoncule \\
\hline der Horror Vacui & I'horreur du vide \\
\hline die Hepatitis & l'hépatite \\
\hline das Imperium & l'empire \\
\hline das Kriterium & le critère (en sport: le critérium) \\
\hline das Neolithikum & le néolithique \\
\hline der Nuntius & le nonce \\
\hline die Plebs & la plèbe \\
\hline das Säkulum & le siècle \\
\hline der Sympathikus & le sympathique \\
\hline die Tabula rasa & la table rase \\
\hline die Unio mystica & l'union mystique \\
\hline der Zirkus & le cirque \\
\hline probat & éprouvé \\
\hline in flagranti & en flagrant délit \\
\hline in natura & en nature (également: en vrai) \\
\hline in toto & en totalité (également : complètement, au complet) \\
\hline manu propria & de mes / tes / ses... propres mains \\
\hline per definitionem & par définition \\
\hline per se & en soi, de soi \\
\hline pro memoria & pour mémoire \\
\hline quod erat demonstrandum & c.q.f.d. (= ce qu'il fallait démontrer) \\
\hline realiter & réellement \\
\hline sub specie aeternitatis & face à l'éternité \\
\hline
\end{tabular}

etc. 


\section{Remarques}

1. Certains mots allemands sont des créations récentes (faux latinismes) : das Faszinosum (la chose fascinante), das Kuriosum (la curiosité, la chose curieuse, le fait curieux), die Zellulitis I Cellulitis (la cellulite), der Studiosus (l'étudiant).

2. Dans la condition sine qua non, seul le nom a été francisé.

3. À der Emeritus correspond le professeur émérite.

4. Certains noms ont en allemand également d'autres sens que celui qu'ils ont en français : der Korpus signifie non seulement le corpus, mais le corps (d'un meuble, d'un instrument de musique), le Christ en croix.

5. À die Majestas correspondent le Christ en majesté, la Vierge en majesté.

6. À l'expression ad interim, employée essentiellement en Suisse alémanique, correspond le français par intérim.

\section{Mots ou expressions ayant des sens ou des emplois différents dans les deux langues (faux amis)}

\begin{tabular}{|l|l|}
\hline der Abusus (l'abus) & $\begin{array}{l}\text { l'abusus (droit de vendre, de transformer ou de } \\
\text { détruire le bien dont on est propriétaire) }\end{array}$ \\
\hline $\begin{array}{l}\text { das Curriculum } \\
\text { (le programme [d'enseignement]) }\end{array}$ & le curriculum (vitae) (der Lebenslauf) \\
\hline $\begin{array}{l}\text { der Kurator (l'administrateur, le commissaire } \\
\text { [d'exposition], le conseiller [université] }\end{array}$ & le curateur (der Vormund, der Betreuer) \\
\hline der Kursus (le cours) & le cursus (der Studiengang ; die Laufbahn) \\
\hline $\begin{array}{l}\text { das Pensum (la tâche ; le programme, } \\
\text { les contenus d'enseignement) }\end{array}$ & le pensum (die Strafarbeit, die Pflichtarbeit) \\
\hline die Ratio (la raison) & le ratio (das Verhältnis) \\
\hline der Usus (la coutume) & l'usus (das Benutzungsrecht) \\
\hline der Terminus (le terme technique) & le terminus (die Endstation) \\
\hline $\begin{array}{l}\text { das Votum (la voix, la décision, la prise de } \\
\text { position ; (CH) l'intervention - } \\
\text { dans un parlement, une assemblée) }\end{array}$ & $\begin{array}{l}\text { le vote (die Abstimmung; die Verabschiedung, } \\
\text { die Bewilligung ; die Wahl) }\end{array}$ \\
\hline post partum (après l'accouchement) & le post-partum (die Zeit nach der Entbindung) \\
\hline ein gewisser Quidam (veraltet) & un quidam \\
\hline $\begin{array}{l}\text { ad hoc (Adv. dans ce cas précis ; } \\
\text { immédiatement, spontanément) }\end{array}$ & ad hoc (Adj. passend) \\
\hline ex aequo (de la même manière) & ex aequo (gleichstehend) \\
\hline in extremis (à l'agonie) & in extremis (in letzter Minute) \\
\hline in petto haben (avoir en réserve) & in petto (insgeheim, im Stillen) \\
\hline notabene (il faut le souligner) & le nota bene (die Anmerkung) \\
\hline
\end{tabular}

etc. 


\section{Mots latins en allemand et mots tout à fait différents en français (faux amis)}

\begin{tabular}{|l|l|}
\hline die Crux/Krux & le problème, le hic (fam.) \\
\hline der Exitus & la mort \\
\hline die Fama & la réputation \\
\hline die Fata Morgana & le mirage, l'apparition, la vision \\
\hline der Kustos & le conservateur \\
\hline die Laudatio & l'éloge, le discours (à la louange de) \\
\hline der Lokus (ugs.) & le petit coin (fam.) \\
\hline das Omen & le présage \\
\hline der Ordinarius & le professeur (titulaire) \\
\hline der Orkus & les Enfers \\
\hline das Plazet & l'accord, l'assentiment \\
\hline die Suada & le flot de paroles, la grandiloquence, la volubilité \\
\hline das Suspensorium & la coquille (boxe et autres sports de combat) \\
\hline der Turnus & le roulement \\
\hline die Ultima Ratio & le dernier recours (''ultima ratio = das letzte Argument) \\
\hline die Vita & la biographie \\
\hline ad absurdum führen & prouver l'absurdité de \\
\hline ad acta legen & classer \\
\hline anno (+ Datum) & en (+ date) \\
\hline Anno Domini (+ Datum) & en l'an de grâce (+ date) \\
\hline coram publico & en public \\
\hline ceteris paribus & toutes proportions gardées \\
\hline cui bono ? & à qui cela profite-t-il ? \\
\hline circa/zirka & environ \\
\hline cum grano salis & toutes proportions gardées \\
\hline eo ipso & naturellement, de soi-même \\
\hline horribile dictu & c'est terrible de devoir dire cela \\
\hline idealiter & dans le meilleur des cas \\
\hline in corpore & au complet (cf. remarque 2) \\
\hline in dubio pro reo & le doute profite à l'accusé \\
\hline in nuce & brièvement, essentiellement \\
\hline
\end{tabular}




\begin{tabular}{|l|l|}
\hline in puncto... & en ce qui concerne... \\
\hline in spe & futur (adjectif antéposé) \\
\hline in statu nascendi & au stade embryonnaire \\
\hline in summa & en tout \\
\hline in praxi & en réalité, effectivement \\
\hline etwas intus haben (ugs.) & avoir descendu (= bu) qqch. (fam.) \\
\hline nomen est omen & il/elle porte un nom prédestiné \\
\hline post festum & après coup \\
\hline postnumerando & après coup, à terme échu \\
\hline praenumerando & d'avance, par anticipation \\
\hline prima facie & à première vue \\
\hline privatim & en privé, confidentiellement \\
\hline qua & à travers, au moyen de, selon \\
\hline sine tempore & ponctuellement \\
\hline sit venia verbo & qu'on me pardonne l'expression \\
\hline summa cum laude & avec mention très honorable \\
\hline summa summarum & en tout, tout compris \\
\hline
\end{tabular}

etc.

\section{Remarques}

1. Quelques faux latinismes relèvent du langage estudiantin : der Luftikus (l'individu insouciant, superficiel), der Pfiffikus (le petit malin, le débrouillard), der Fidibus (petite torche de papier plié servant notamment à allumer une pipe).

2. En français, in corpore est un helvétisme : le gouvernement in corpore. 


\section{Mots latins en français et mots tout différents en allemand (faux amis)}

\begin{tabular}{|c|c|}
\hline le distinguo & die Unterscheidung \\
\hline le lupanar & das Freudenhaus \\
\hline le mea culpa ; faire son mea culpa & das Schuldbekenntnis ; sich schuldig bekennen \\
\hline le médius & der Mittelfinger \\
\hline le minus (habens) & die Niete, die Null, der Versager \\
\hline l'occiput & der Hinterkopf \\
\hline l'olibrius & der komische Kauz \\
\hline le pretium doloris & der Schadenersatz \\
\hline le satisfecit & die Genugtuung \\
\hline le summum & der Gipfel, der Höhepunkt \\
\hline le verbatim & die wortwörtliche Niederschrift \\
\hline le vulgum pecus & die Normalsterblichen \\
\hline l'ut & $\operatorname{das} C$ \\
\hline envoyer/expédier ad patres (fam.) & kaltmachen, den Garaus machen (fam.) \\
\hline ad vitam aeternam & ewig \\
\hline faire chorus & sich anschließen \\
\hline de visu & durch persönlichen Augenschein \\
\hline X dixit & Originalton $X$ \\
\hline exit (indication scénique) & geht $a b$ \\
\hline ex nihilo & aus dem Nichts \\
\hline grosso modo & in groben Zügen, ungefähr \\
\hline horresco referens & ich schaudere beim Erzählen \\
\hline illico (fam.) & sofort, auf der Stelle \\
\hline in fine & letztendlich \\
\hline lato sensu & im weiteren Sinne \\
\hline manu militari & mit Gewalt \\
\hline mordicus (fam.) & steif und fest \\
\hline motus (et bouche cousue)! (fam.) & nichts verraten! \\
\hline primo, secundo, tertio & erstens, zweitens, drittens \\
\hline quid de... ? & wie steht es um...? \\
\hline rasibus (fam.) & ganz knapp, ganz dicht \\
\hline sine die & auf unbestimmte Zeit \\
\hline stricto sensu & genau genommen \\
\hline
\end{tabular}

etc. 


\section{Remarques}

1. Au gratis pro deo (fam.) français correspond le gratis allemand.

2. Rasibus (fam.) et motus (fam.) sont de faux latinismes.

En guise de conclusion, une évidence : en matière d'emprunts aussi bien que de manière générale, chaque fois qu'on se trouve en présence d'une ressemblance formelle, il convient de se méfier et de vérifier les définitions et les emplois dans les dictionnaires monolingues. Regrettons au passage que les dictionnaires bilingues - PONS, Langenscheidt, Larousse présentent autant de lacunes.

Michel CORBELLARI est licencié en allemand et en français. II a enseigné ces deux langues dans un collège et au lycée de La Chaux-de-Fonds (Suisse). Parallèlement, il a enseigné la didactique du français à l'Institut de formation des maitres du canton de Neuchâtel. En outre, il est coauteur de divers manuels de français destinés au premier cycle du secondaire. 